\title{
GUERRA, ESTADO Y PAZ UNA CONTINUACIÓN DEL PENSAMIENTO DE CLAUSEWITZ ${ }^{1}$
}

\author{
ANDERS BOSERUP \\ con un preámbulo de Klaus Schriewer (Universidad de Murcia) \\ y Ángel Guirao Navarro (Universidad de Tubinga y Universidad Doshisha)
}

\begin{abstract}
RESUMEN: El artículo, partiendo del pensamiento de Carl von Clausewitz, elabora y defiende la hipótesis de que la lucha de reconocimiento entre los Estados y la superioridad de la defensa exigen tomar al Estado como sujeto sui generis y, por lo tanto, como punto de partida de la teoría social y del pensamiento sobre sociedad y cultura. En la introducción se delimita el campo de debate y se determinan los rasgos del trabajo científico. En el capítulo primero se indagan las implicaciones del concepto clausewitziano de guerra. El segundo profundiza en el concepto de pausa, con objeto de poder dibujar, trazar las bases teóricas de defensa y ofensiva tratadas en el tercer capítulo. El último capítulo expone los rasgos generales del concepto Estado como un sujeto que se encuentra en una lucha permanente de reconocimiento. El artículo fue publicado en 1990 en alemán y se presenta aquí por primera vez en castellano.
\end{abstract}

PALABRAS CLAVE: Estado, guerra, teoría social, Clausewitz.

\section{War, State and Peace. A continuation of Clausewitz' thinking}

ABSTRACT: The article, which parts from the thoughts of Carl von Clausewitz, works out and defends the hypothesis that the struggle for recognition among states, and the superiority of the defense, demands to understand the state as a sui generis subject. Therefore it should be taken as a starting point for social and cultural theory. The introduction describes the field of debate and determines the characteristics of scientific work. The first chapter deals with the implications of Clausewitz'concept of war. The second chapter delves into the concept of pause aiming to be able to work out the theoretic bases of defense and attack in the third chapter. The last chapter points out the general characteristics of the state-concept as a subject in permanent struggle for recognition. The article was published in German in 1990 and is now presented for the first time in Spanish.

KEY WORDS: State, war, social theory, Clausewitz.

\section{Preámbulo de los traductores}

Presentamos aquí la traducción de un texto publicado en 1990 por el pensador danés Anders Boserup (1940-1990) cuya reflexión sobre la lucha de reconocimiento, la guerra y el Estado es de suma importancia para las Ciencias Sociales y la Filosofía política. Lo es porque anima con buenos argumentos a un cambio paradigmático en el pensamiento y la investigación sobre la guerra, el Estado, la sociedad y

1 Traducción del alemán de Klaus Schriewer y Ángel Guirao Navarro de: Boserup, AnDERs: «Krieg, Staat und Frieden. Eine Weiterführung der Gedanken von Clausewitz», aparecido en: Weizäcker, Carl Friedrich von (ed.), Die Zukunft des Friedens in Europa. Politische und militärische Voraussetzungen, Hanser, München y Wien 1990, pp. 244-263. Aunque Pensamiento tiene como norma publicar sólo textos originales, se ha decidido hacer en este caso una excepción para dar a conocer este texto en español. 
las culturas de los diferentes grupos sociales. Propone dejar en un segundo plano la tradición hobbesiana que construye al Estado desde dentro, partiendo de la idea de un contrato social. En su lugar elabora las bases de una concepción teórica que toma la relación entre los Estados como punto de partida de su desarrollo.

Boserup encuentra la inspiración para su propuesta en el teórico de la guerra Carl von Clausewitz. Invita a una revisión de la interpretación de su obra Sobre la guerra y la utiliza como punto de partida para el desarrollo de un concepto renovado de Estado, al cual define como un sujeto que se encuentra en una lucha de reconocimiento permanente en el sistema interestatal. Sobrevivir en él exige que el Estado sea capaz de generar ciertos recursos y, en su caso, activarlos de una manera que obliga a los otros Estados a reconocer su soberanía sobre el territorio y la población que le pertenecen. Esta labor de soberanía hacia el exterior hace necesario que el Estado sea también capaz de organizarse interiormente de una manera que permita la creación de estos recursos.

Esta lectura de Clausewitz no se apoya en el supuesto vínculo con el filósofo alemán J. G. Fichte que ha postulado José Luis Villacañas Berlanga ${ }^{2}$, sino que sigue la tesis defendida por varios autores que señalan la influencia clave de Hegel en esta obra $^{3}$. Boserup identifica las figuras del pensamiento hegeliano y especialmente los conceptos praxis y lucha de reconocimiento como claves para entender la perspectiva que nos abre este pensador de la guerra a la hora de desarrollar una teoría de lo político, social y cultural que parte del Estado como un sujeto superior en la historia humana.

El texto cuya traducción presentamos aquí fue escrito por Anders Boserup un año antes de su muerte en 1990. Fue publicado en su primera y única edición en alemán ${ }^{4}$ y ahora se ha traducido al español. Es uno de los pocos textos de este autor, que siempre dudaba en publicar porque siempre le pareció que sus escritos podrían mejorarse. Su empeño por la perfección transluce con solo considerar la historia del propio texto. Boserup había elaborado el manuscrito original en danés y pidió a tres colaboradores alemanes (entre ellos Klaus Schriewer) traducir el texto al alemán. Después de recibir y leer la versión alemana, pidió revisar el texto en común. Se reunió con sus colaboradores, que esperaban una reunión de algunas horas, pero en realidad la revisión del texto tardó nada menos que catorce días a jornada completa. Se habló de cada palabra y sus significados, comparando cada término con posibles sinónimos e incluso con palabras de otros idiomas con objeto de acercarse lo más posible a la intención que quería trasmitir el autor. Así se ha elaborado un texto que en cada una de sus líneas ha sido pensado al máximo. La

2 Villacañas Berlanga, José Luis, La Nación y la Guerra. Confederación y hegemonía como formas de concebir Europa, Editum, Murcia 1999.

3 Courmier, Youri: «Hegel and Clausewitz: Convergence on Method, Divergence on Ethics», The International History Review, Volume 36, Issue 3, 2014, pp. 419-442. HerberG-Rothe, Andreas. "Clausewitz und Hegel. Ein heuristischer Vergleich», Forschungen zur brandenburgischen und preußischen Geschichte. Jahrgang 10, Heft 1/2000, pp. 49-84. Rothe, Barbara and TürPE, André, «Das Wesen des Krieges bei Hegel und Clausewitz», Deutsche Zeitschrift für Philosophie 25, 1977, pp. 1331-1343.

4 Boserup, Anders, «Krieg, Staat und Frieden. Eine Weiterführung der Gedanken von Clausewitz», en: Weizäcker, Carl Friedrich von (ed.), Die Zukunft des Friedens in Europa. Politische und militärische Voraussetzungen, Hanser, München y Wien 1990, pp. 244-263. 
traducción española intenta recoger con la mayor precisión posible las ideas descritas, sabiendo que la transformación del alemán al español siempre lleva consigo ciertas dificultades.

A modo de indicación desearíamos reseñar que precisamente para ahorrarle dificultades añadidas al lector, adjuntamos al final una bibliografía tanto de las obras que citamos en calidad de traductores, así como de las obras citadas por Boserup. Persiguiendo la misma finalidad hemos completado las citas de Boserup y localizado los textos en castellano.

En el mundo hispanohablante, Boserup es conocido por su libro Guerra sin Armas, escrito conjuntamente con el británico Andrew Mack en 19745 ${ }^{5}$, traducido al español en 1985 y reeditado en $2001^{6}$. Este libro, que versa sobre diferentes experiencias históricas de defensas no-violentas, contiene una primera alusión a la obra de Clausewitz cuyo análisis se desarrolla en profundidad en el artículo presente.

Universidad de Murcia,

KLAUS SCHIEWER

ks@um.es

Universidad de Tubinga (Alemania) y

Ángel Guirao Navarro

Universidad de Doshisha (Japón),

aguiraonavarro101@hotmail.com

\section{GUERRA, ESTADO Y PAZ. UNA CONTINUACIÓN DEL PENSAMIENTO DE CLAUSEWITZ}

\section{INTRODUCCIÓN}

La guerra es un medio, un mero medio y sería ilógico pensar que un medio pueda fijar su propio objetivo. El objetivo debe ser determinado en otro lugar. Este otro lugar se llama política. La política, por lo tanto, es provisionalmente solo un otro lugar en relación con la guerra, que de una forma no especificada establece el objetivo de la guerra. Clausewitz no se refiere a otra cosa cuando dice que la guerra es el instrumento de la política.

Clausewitz trata aquí el aspecto subjetivo de la política, política como voluntad, como «inteligencia del Estado personificado» que estimula el instrumento. Aquí la política es solamente el nombre para el lugar donde se determina el

\footnotetext{
5 Boserup, Anders; MACK, Andrew, War without weapons. Non-Violence in National Defense, Schocken Books, New York 1974.

6 Boserup, Anders; Мack, Andrew, Guerra sin Armas. La noviolencia en la defensa nacional, Editorial Fontamara, Barcelona 1985. Segunda edición: Libros de la Catarata, Madrid 2001.
} 
objetivo. La teoría requiere necesariamente un lugar semejante, pero no puede decir nada sobre su naturaleza. Si una teoría de la guerra quisiera manifestarse sobre el contenido de la voluntad política, no cumpliría con sus propios presupuestos. En una cadena de objetivo - medio, la finalidad necesariamente es libre e indeterminada.

Sin embargo, Clausewitz emplea el termino política también en otro sentido, un sentido más objetivo, que no pertenece a su figura teórica principal. Con él describe las relaciones sociales e internacionales concretas, de los cuales resulta la guerra y que están sujetas a él. La política — tomada en este sentidodetermina el carácter cambiante de la guerra, porque las condiciones históricas dadas fijan los limites para la capacidad, la forma y la aplicación de los instrumentos militares.

El análisis teórico de Clausewitz considera la guerra en sí: qué formas asume, qué objetivos parciales se fija y cómo aplica los medios en relación con los objetivos. En este contexto, Estado, política y sociedad son solamente el marco externo del análisis dado desde el principio que fija la finalidad de la guerra, determina sus medios, la provoca y controla su desarrollo. Las variaciones de estos marcos influyen, tal y como subraya Clausewitz, en la guerra, y le dan aspectos cambiantes en diferentes épocas históricas ${ }^{7}$.

Aunque las guerras provengan de circunstancias sociales y en este sentido son continuación y secuela de la política, hay que destacar que la guerra es, por sí misma, una fuerza dominante en el desarrollo histórico. Sistemas sociales e imperios, cuya capacidad de defensa no correspondió a las exigencias de su tiempo, fueron barridos. En última instancia, sin embargo, no sólo es decisiva la Guerra sino también la permanente posibilidad de entrar en guerra. Ella presiona a los Estados permanentemente a realizar reformas sociales y políticas en aras de mantenerse o simplemente para sobrevivir en el escenario internacional. No es sólo en la guerra "actual» y real que "cada uno da a los otros la ley» ${ }^{8}$, sino también sucede en la guerra «virtual» y meramente posible.

Este efecto de la guerra sobre la política del Estado abre la posibilidad de fundamentar una construcción teórica a partir de la comprensión de la guerra que tenía Clausewitz, la cual no solo incluye un concepto de guerra, sino que también está dirigida a un concepto de Estado y de Política. La teoría de la guerra de Clausewitz parece no solo tener interés para la Ciencia Bélica, sino también puede ser un posible punto de partida para otras disciplinas que necesitan urgentemente un nuevo enfoque teórico, como la Investigación de la Paz, la Sociología, las Ciencias Políticas, la Historia y la Política Internacional. Aquí encontramos un amplio campo que en general todavía no está trabajado.

En las siguientes páginas se intentará demostrar algunas de las consecuencias de la teoría de la guerra de Clausewitz para el análisis de la paz, del Estado y su estructura interna así como las relaciones mutuas entre los Estados.

7 Clausewitz, Carl von, De la guerra, Solar, Buenos Aires 1983, libro 8, capítulo 3B.

8 Ibid., libro 1, capítulo 1, parte 3. 
Aunque estas consideraciones solo pueden ser esbozadas aquí, es necesario proceder en el desarrollo del concepto con cuidado y seguir de cerca cómo y en qué orden están construidos los distintos conceptos. Y es que la teoría de Clausewitz trabaja - como cualquier verdadera teoría - con conceptos, que no son denominaciones para observaciones, sino entramados de ideas cuidadosamente construidos, que recogen sus contenidos significativos de las relaciones con otros conceptos. Es por esta forma tan específica de interconexiones lógicas de los conceptos que es posible deducir relaciones necesarias, esto es, leyes científicas. Por eso una teoría verdadera no es un marco abierto de interpretación, no es una perspectiva que acepte cualquier complementación. La teoría construye un sistema coherente de conceptos y relaciones de conceptos: por ello tiene valor explicativo real y, por ello, se pone a prueba en su totalidad cuando se aplica a la realidad.

El desarrollo de una teoría es un proceso gradual de especificación del sistema conceptual, que va desde las formas teóricas abstractas más sencillas hasta las más complejas. Este proceso de especificación está influido naturalmente por representaciones y conocimientos sobre «la realidad». Pero es importante recordar una y otra vez que los sistemas conceptuales derivados posteriormente no son más que modificaciones y ampliaciones de los anteriores y que, al igual que estos primeros, son puros sistemas conceptuales. El proceso de especificación de la teoría no comienza en lo teórico para luego ir acercándose paulatinamente a «la realidad». Nunca se abandona el plano teórico. Fuera del plano teórico solo hay descripciones históricas de ejemplos reales, que sólo pueden servir como banco de pruebas.

\section{LA GUERRA}

El punto de partida es el concepto de guerra de Clausewitz. Para nuestros propósitos es muy útil presentarlo como una especificación gradual de tres formas teóricas, que aquí denominamos "guerra explosiva», "guerra virtual» y "guerra moderada». No es la intención seguir a Clausewitz paso a paso, sino destacar aspectos de su teoría que en nuestro contexto son significativos. Debe ser subrayado que estas tres formas teóricas efectivamente están emparentadas con las inculpadas categorías de «guerra-absoluta» y "guerra-limitada», pero no deben ser confundidos con ellos.

La primera forma teórica, la guerra explosiva, la describe Clausewitz en las primeras páginas de su obra De la guerra. Aquí trata en primer lugar sobre la relación polar entre los enemigos y la racionalidad de la meta de los actos de guerra. La política define la finalidad de la guerra. Pero tal finalidad es desplazada por el objetivo de la guerra: conseguir la victoria, evitar la derrota. La guerra es, por lo tanto, definida desde el inicio como una actividad guiada por un objetivo entre dos adversarios, en el cual los objetivos tienen un carácter especial: son singulares (solo hay un único objetivo: la victoria), son universales (en todas las 
guerras se persigue el mismo objetivo) y son contrapuestos de manera polar (la victoria de uno es la derrota del otro).

La singularidad del objetivo asegura que todos los elementos de las guerras estén interrelacionados en una totalidad jerárquica y ordenada y que la estrategia se transforme en un cálculo unívoco —en contraposición con la ponderación «subjetiva» de las distintas consideraciones de la Política-.

La universalidad del objetivo permite la construcción de una teoría de la guerra como un fenómeno universal, a pesar de todas las diferencias históricas.

De la polaridad del objetivo Clausewitz deduce una interrelación entre los adversarios que provoca que la guerra, en esta primera forma de la teoría, sea explosiva y sin límites. Cada uno de los adversarios debe temer que el enemigo va a aprovechar cualquier circunspección, porque éste persigue sin miramientos la victoria, y por su parte causa el miedo a su rival, lo que explica que no se atreva a comportarse de forma distinta. Esta interrelación se impone a ambas partes de forma objetiva y arranca de cada uno lo más extremo de sus fuerzas.

Este sistema conceptual tan sumamente sencillo recoge las principales características de la guerra. A pesar de ello no corresponde en muchos casos con la experiencia: las guerras reales no discurren en una única descarga ininterrumpida de la fuerza. A menudo ambas partes parecen mantenerse tranquilas y no emprender nada.

Por ello, la siguiente forma teórica de guerra, la guerra virtual, es una modificación del concepto de guerra explosiva, la cual debe justificar desde una perspectiva teórica la aparición de la inacción en la guerra. Para ello, es necesaria una especificación conceptual que supere la polaridad absoluta entre los enemigos. Con esta finalidad, Clausewitz introduce la distinción entre ataque y defensa. Se trata de formas de lucha con respecto a las cuales los contrincantes se comportan de forma opuesta (cuando el uno ataca, el otro debe necesariamente defenderse y a la inversa), pero no son polares puesto que no tienen por qué ser igualmente fuertes. Mientras que al atacante le gustaría retardar su ataque, por ejemplo para acercar más tropas, el atacado debe querer por la misma razón que el ataque se produzca más pronto que tarde. En cuanto a los objetivos, rige por lo tanto la total polaridad. Pero de lo anterior no se deduce que la otra parte que quiere ser atacada mejor antes que después, sea capaz por su parte de atacar. Lo que le concierne a los medios no rige por lo tanto ninguna polaridad completa. Si se acepta además que el ataque es más difícil de llevar a cabo que la defensa (esta tesis se justifica más adelante), es igualmente posible para ambas partes que vean como interés propio esperar.

La posibilidad de una pausa en la guerra depende por ello de que la forma de defensa — la que es la forma de lucha que espera y detiene- sea más fuerte que la forma de ataque.

Cuanto más destacada sea la superioridad de la defensa, tanto más estampará la pausa a la guerra. Mientras que la guerra se encuentre en pausa, puede ser adjetivada como guerra virtual. La contienda se encuentra interrumpida, pero la guerra continúa en cierto sentido, es decir, como una posibilidad constante, 
la cual simplemente no será actualizada mientras las condiciones de la pausa puedan mantenerse. La pausa es así sólo un precario y temporal equilibrio, y depende de que las fuerzas que se oponen se mantengan constantemente a raya. Y ello a pesar de que incluso cuando la guerra solo sea «virtual», sigue acuciando de forma real a los oponentes.

En la tercera forma teórica de la guerra, la guerra moderada, aparecen los motivos políticos de una manera más directa. Durante la pausa (cuando ninguna parte es suficientemente fuerte para forzar una decisión, pero tampoco la tiene que temer), la guerra puede adquirir formas más limitadas, cuando los adversarios solo intentan pequeñas ganancias que se presentan mientras que ambos se encuentran por así decirlo a la defensiva. Al abrigo de la pausa se ofrece margen de maniobra para la guerra ni hacia un lado ni hacia el otro, con débiles motivos y modestas expectativas que solamente pueden poner en marcha fuerzas limitadas. Por ello hay guerras de distinta intensidad; guerras que tienden a la completa aniquilación del enemigo, guerras que únicamente persiguen objetivos limitados, y guerras que apenas pasan de ser un recíproco control armado.

Algunos de los pasajes de la obra de Clausewitz podrían dar la impresión de que la debilidad de algunas guerras puede ser explicada adecuadamente por medio de nimios e irrisorios motivos, puesto que sólo pueden poner en marcha fuerzas modestas. En el primer libro, capítulo 1, apartado 11 por ejemplo, puede leerse así, si se desatiende que las siguientes páginas deben mostrar que las guerras moderadas no pueden encontrar ningún final pero deberían ser llevados más allá de lo intencionado, "por lo que vendría una continuidad en la acción militar que lo potenciaría todo de nuevo».9

La guerra moderada no puede pensarse entonces sin la guerra explosiva y la guerra virtual. Clausewitz también la define como una modificación, y no como algo independiente..$^{10}$ En la guerra moderada, la guerra explosiva es una condición indispensable. Sólo cuando las fuerzas y motivos fuertes se mantienen a raya (y no es que simplemente se encuentren ausentes), pueden entrar en juego los objetivos más limitados y las acciones más ordenadas, «porque ya no se encontrarán por más tiempo bajo la presión de una gran decisión ni tampoco de un gran peligro ${ }^{11}$. Solo cuando todo está en equilibrio pueden entra en juego las «fichas» más pequeñas, y es imposible imaginarse que puedan jugar libres y aportar beneficios sino en el área conflictiva, que se genera por la parálisis de las grandes fuerzas y motivos.

Es necesario destacar esta relación, porque la guerra explosiva y la moderada a menudo son contrapuestas como «absoluta» y «limitada», como si de cosas distintas se tratase. Esta es la famosa tesis del doble tipo de guerras, cuyo más importante intercesor en los últimos años ha sido Raymond Aron (1976). Su punto de partida es la asunción de dos diferentes teorías de la guerra en

$9 \quad$ Ibid., libro 1, capítulo 1, parte 14.

10 Ibid., libro 8, capítulo 3A.

11 Ibid., libro 6, capítulo 30. 
Clausewitz: una sobre la guerra «absoluta» (que tiene por objetivo la aniquilación), y otra sobre la guerra «limitada» (la que meramente consiste en una vigilancia armada mutua). Tal adopción puede llevar a la conclusión de que la guerra explosiva es «mera teoría», que es inservible en la era nuclear, mientras que la guerra «limitada» debe ser la forma «real». Ya se ha indicado arriba hacia la principal insubsistencia de la diferenciación entre teorías que se aproximan más o menos a la realidad. Aún peor es cuando la relación entre ambos conceptos teóricos se interrumpe. Porque entonces ya no se puede explicar desde una perspectiva teórica la limitación de la guerra, con la consecuencia de que toda la teoría de Clausewitz se convierte el algo inaplicable para la comprensión del problema de la guerra. ${ }^{12}$

\section{LA PAUSA}

Si la defensa es superior a la ofensiva, se puede pensar la paralización en la guerra como el efecto de una situación en que las fuerzas se tienen en jaque. Este concepto de pausa es pensado en Clausewitz para la guerra, pero se puede ampliar hasta un concepto de paz que entiende la paz como una larga interrupción, como un tipo de armisticio permanente. En esta paz, la superioridad de la defensa no solo reprime el acto bélico sino también limita las ambiciones y temores de ambas partes. Cuando todos son más débiles en el ataque que en la defensa, puede ocurrir que la totalidad de los Estados en un sistema de Estados sean suficientemente fuertes para impedir el ataque de otro, pero al mismo tiempo son demasiado débiles como para atacar por sí mismos.

Esta paz (dicho con más detalle: esta estabilidad militar dentro de un sistema de Estados) se basa completamente en el supuesto de que la forma bélica de defensa sea superior. Cuanto mayor sea la superioridad, más robusta será la paz. Hay que subrayar enfatizando, que la igualdad de las fuerzas opuestas, que se suele considerar como requisito previo para la estabilidad militar, es completamente insignificante - excepto cuando la relación de fuerzas es tan notablemente desigual, que la parte débil, aun cuando se aprovecha totalmente de las ventajas de la defensa, no pueda estar segura. Este concepto de paz deducido del pensamiento de Clausewitz no se basa por lo tanto en la igualdad de las fuerzas, sino en un tipo de desigualdad de la fuerza, es decir, en la desigual fuerza del ataque y la defensa.

Si imaginamos por un momento, que la ofensiva y no la defensa fuera la fuerza bélica superior, el mundo sería completamente distinto a como actualmente es. En una situación tensa entre dos Estados debe cada uno de ellos buscar el primer ataque. La historia presentaría un estado de guerra permanente

12 Como Raymond Aron mismo reconoce, sólo queda una «rivalidad» armada que sólo se limita por la «inteligencia política-estratégica» del adversario. ARon, Raymond, Penser la guerre. Clausewitz, Editions Gallimard, Paris 1976, p. 544. 
que solo encontraría un final, cuando todo poco a poco fuera integrado en un imperio global, o cuando todo finalmente sucumbiese. En el razonamiento de Clausewitz, el sistema de muchos Estados, que ha sobrevivido entre la guerra y la paz durante siglos, es la consecuencia directa y la prueba histórica indirecta de la superioridad de la defensa. Esto muestra de nuevo que la paz en el sistema de Estados se basa en la superioridad de la defensa y no en cualquier igualdad de potenciales militares, que supuestamente nunca existió. La igualdad de las fuerzas es mucho más compatible con un estado continuo de guerras.

El Principio de la superioridad de la defensa evidentemente no afirma que los Estados en defensa consigan normalmente (o alguna vez) la victoria. La defensa como forma bélica es superior en principio. Da mejores perspectivas para la victoria que la ofensiva. Sin embargo, puede ser que un Estado, aun cuando se defienda, sea demasiado débil e incluso cometa todo tipo de fallos, lo que le llevaría a desaprovechar esa superioridad.

No obstante, contradice el pensamiento corriente considerar la paz simplemente como una pausa de la guerra. Nos hemos acostumbrado a ver la relación invertida, considerar la paz como la «situación normal» entre los Estados, y la guerra como el fracaso del sistema, como interrupción de las relaciones sujetas a normas entre los Estados.

Una perspectiva no es más correcta que otra. Una perspectiva teórica nunca es verdadera o falsa, sino más o menos fructífera en determinadas relaciones teóricas o prácticas.

Contemplar la guerra (tal y como ella se nos aparece directamente) como un derrumbamiento temporal de un sistema de Estados significa que se excluye toda comprensión de las condiciones de la paz, así como de la naturaleza de la guerra. Entonces, no serán comprensibles ni la racionalidad «interna» de la guerra, esto es, la racionalidad de la meta y con ello los principios generales de la estrategia; ni tampoco su racionalidad «externa», esto es, la racionalidad del fin como refugio último de los Estados. Si la Guerra fuera simplemente el fracaso de un sistema, simple anarquía, entonces resultaría evidente que cualquier teoría con respecto a ella sería imposible. El por qué tal anarquía tendría que ser la última solución de los Estados para imponer su voluntad quedaría siempre como un enigma.

Lo único que queda en esta situación es la representación de la guerra como una cosa amorfa. O bien es uno puro derribo, o una continuación de la relación política de los Estados añadiendo a ésta medios violentos, pero sin que pueda ser fijado ningún principio, como una estrategia que pueda administrar esta combinación de medios políticos y militares. Entonces se está, nuevamente, en un vacío teórico, que se observó ya en relación con la tesis de la doble forma de la guerra. Se dejará en manos de una «razón política» no definida con más detalle, llenar este vacío lo mejor posible. No se trata solamente del concepto de guerra sino también del concepto de paz que queda en el sinsentido. 


\section{LA DEFENSA}

La argumentación de Clausewitz respecto a la hipótesis de la superioridad de la defensa no vamos a repetirla aquí debido a su extensión. Él trata este tema en todo el libro 6 y 7 , siendo más de un tercio de la obra.

A pesar de ello su descripción no es satisfactoria. Aunque la diferencia entre ataque y defensa se introduce precisamente allí donde es necesaria, a saber, al hilo de la explicación de la pausa, ${ }^{13}$ no se dice nunca dónde tiene su razón en la construcción teórica. Clausewitz no muestra en ninguna parte que esta separación entre ambas formas de estrategia bélica se deduzca necesariamente de la naturaleza de la guerra. Por ello no se llega a esclarecer por qué la guerra se divide de esa manera, por qué en dos formas, por qué solamente estas dos formas, y finalmente en qué consiste la diferencia realmente. No es suficiente que nosotros podamos aplaudir reconociendo, cuando esta denominación (en el libro 1) se presenta por primera vez, y que se pretenderá explicar poco a poco (en el libro 6), qué es lo que se quiere señalar. Mientras que la defensa y el ataque no sean definidos como conceptos que se hallan en relación el uno con el otro, y con los restantes conceptos de la teoría, no se podrá por desgracia demostrar que se encuentran relacionadas específica y necesariamente: que son contrarios, aunque sin ser mutuos reflejos, y que la superioridad de la defensa es realmente universal e indispensable, y no se basa en condiciones (por ejemplo de tipo tecnológico) que no siempre están presentes.

Para encontrar la base de la diferencia debe recurrirse al concepto de "praxis». Este concepto trata de la intervención teleológica de un sujeto frente a un objeto, que se considera como algo inanimado, como una simple cosa. El concepto de praxis contiene por una parte una relación finalista entre objetivo y medio, y por otra parte contiene una relación causal entre medio y objeto. El medio es por consiguiente vínculo entre la parte «ideal» y «material» de la relación de la praxis. Resulta obvio que Clausewitz presupone implícito tal concepto de «praxis» cuando piensa la guerra desde el comienzo como un acto de violencia, en la que la violencia física sirve como un medio para imponer su voluntad al enemigo. Antes incluso de que la guerra llegue a ser bilateral, la acción militar es parte de una praxis unilateral, una intervención teleológica física de un sujeto frente a un objeto.

Esta claro que la diferencia entre el ataque y la defensa tiene su origen aquí, en el concepto de praxis, en el que el enemigo todavía solo es pensado como un objeto, como una masa inanimada. Ya aquí están a la vista las principales características destacadas por Clausewitz: el ataque es la forma activa con fin concreto, la defensa la que está a la espera, la que defiende. En su forma más simple, la defensa es solo la resistencia pasiva, inanimada, la «inercia» del objeto.

Clausewitz considera que una fuerza viva que actúe sobre una masa muerta no es una guerra. Esto aquí no debe negarse. La guerra siempre consta de dos

13 Clausewitz, Carl von, De la guerra, Solar, Buenos Aires 1983, libro 1, capítulo 1, parte 16. 
fuerzas vivas, las cuales chocan entre sí, ${ }^{14}$ porque solo con la intervención activa del adversario al defenderse, cuando éste se transforma de un objeto en un oponente, la guerra será algo más que simple praxis - pues se convierte en un conflicto estratégico, en un choque de dos voluntades. Pero es importante tener en cuenta que se debe volver a un punto, que tiene su lugar en la lógica de la teoría todavía antes de la primera determinación de la guerra como duelo, si se quiere entender que son el ataque y la defensa en el fondo - aunque así por el momento solo se consigue un concepto empobrecido de la defensa.

Si Rusia en 1812 se hubiera abstenido de cada operación militar directa permitiendo que el frío del invierno y las dimensiones del país desgasten las fuerzas napoleónicas y les obligasen a la retirada, sería esto un ejemplo de la forma bélica sin enemigo que consiste solamente en la praxis del atacante contra una masa muerta.

La defensa en esta forma sin enemigo se comporta como si tuviera a disposición medios bélicos, a saber, aquella masa que aunque carente de vida es efectiva como resistencia. El ataque por su parte dispone de fuerzas «vivas», es decir, fuerzas dirigidas teleológicamente por una Inteligencia.

Más concretamente se podría decir que la ofensiva dispone de medios movilizables libres, pero también limitados (es decir, el ejército) mientras que la defensa en su forma pura o carece de tales medios o no los aplica. La defensa por su parte posee medios de otro tipo, que a la ofensiva le faltan. Estos medios se movilizan por el avance de la ofensiva y solo pueden entrar en juego de esa manera: las montañas y los ríos del país, la resistencia de la población y el respaldo de otros países, los cuales temen la fuerza futura de un atacante victorioso.

En la guerra, como es en realidad - esto es, cuando la defensa interviene activamente-, el atacante no se encuentra solamente a las montañas y a los ríos, que la naturaleza abúlica había dejado de forma ocasional, sino que también encuentra otras fuerzas «muertas» como murallas y fosos, construidos intencionadamente con el fin de servir como obstáculos para el atacante y como apoyo para el defensor. También estas instalaciones defensivas solo pueden ser transformadas en fuerzas operativas por el avance del atacante. Todas estas fuerzas «atadas» son partes integradas de la guerra. En el ataque se dispone por ello solo de un tipo de fuerzas: las que se pueden movilizar, las fuerzas «vivas», las fuerzas armadas, y nada diferente. En la defensa se dispone tanto de estas fuerzas como de las fuerzas atadas, que se activan por el ataque. A fin y a cabo es esa la asimetría que está detrás de la superioridad de la defensa.

Que la defensa es la forma más fuerte no se deduce por ello del concepto de praxis mismo. La superioridad de la defensa se produce no antes del propio forcejeo, en el cual se combinan fuerzas atadas y libres. De ello también se explica que la defensa no se puede entender como pasividad sino más bien como estar a la espera (excepto en la guerra «sin adversario»). Si fuese solo pasiva

\footnotetext{
14 Ibid., libro 1, capítulo 1, parte 4.
} 
y se conformara solo con las fuerzas atadas, no existiría ningún motivo para suponer que fuera superior al ataque con sus fuerzas libres.

De ello resulta que la regla de la superioridad de la defensa tiene que tener validez universal, aunque dependa de las circunstancias dadas, que dimensión tenga esa diferencia de fuerzas. Hay que advertir especialmente del malentendido que la relación de fuerzas entre defensa y ofensiva cambia a lo largo de la historia, de una manera que a veces la defensa y a veces la ofensiva es la superior. Clausewitz constata de manera seca: «En realidad, el menosprecio de la defensa es una consecuencia de una época, en la cual una manera de defensa es desfasada» ${ }^{15}$.

En cierta medida, la superioridad de la defensa es inagotable. Hasta que la guerra no concluya con una victoria definitiva del ataque sobre todo oponente, cualquier paso de avance del atacante despierta inevitablemente nuevas fuerzas del lado de la defensa.

Tal y como se ha descrito aquí, el ataque y la defensa están contrapuestas, en el preciso sentido que necesitan para cumplir su función teórica en relación con la pausa. Por un lado, la defensa y el ataque no son contrarios polares, porque sus medios son totalmente diferentes. Por otro lado, los adversarios adoptan roles necesariamente opuestos en relación con las formas de guerra: lo hacen por la sencilla razón de que el ataque origina la defensa, no sólo formalmente a modo de «espejo» de si mismo, sino en un sentido material, activando las fuerzas atadas de la defensa. Y por último, por todo lo dicho, es la defensa necesariamente la mas fuerte de estas dos formas de guiar la guerra.

\section{El Estado}

Cuando se quiere estudiar la guerra, tal y como lo hace Clausewitz, se puede partir como marco dado de los Estados y del sistema que estos constituyen. El Estado es entonces actor, y la política su voluntad. La política debe solamente evitar proyectos o intenciones para lo cual no dispone de los suficientes medios. No queda más por decir aparte de esto.

Sin embargo, aquí la tarea es justamente la contraria, esto es, utilizar el concepto de guerra como punto de partida para un análisis mas amplio. Aquí se trata de expandir este concepto de guerra hasta un concepto de Estado, o sea, determinar los rasgos del Estado que se deducen a partir de la naturaleza de la guerra. Se ha comenzado para ello con el concepto de pausa, el cual mostraba cómo la guerra originaba la posibilidad de la paz como estado de equilibrio temporal e inseguro entre los Estados.

En lo que sigue pretendemos acercarnos más a los Estados y su sistema. Por desgracia no podemos llevar a término todas las ideas y consideraciones.

15 Ibid., libro 6, capítulo 2. 
La tarea aquí no es otra que mostrar que una ampliación de los conceptos de Clausewitz es posible, e indicar cómo puede ser emplazada.

Nuestra tarea consiste en continuar lo que Clausewitz ha dejado abierto, puesto que su empresa era otra bien distinta. Una significativa inspiración la da aquí el análisis llevado a cabo por Hegel sobre la lucha por el reconocimiento, así como su análisis sobre la relación entre amo y esclavo, expresada en el capitulo sobre la "Autoconciencia» en la Fenomenología del Espíritu ${ }^{16}$. Esto es evidente porque el análisis hegeliano de la lucha por el reconocimiento y la autoconciencia subyacen a la misma figura lógica que el análisis de la pausa realizado por Clausewitz. Ahora bien, se diferencian por supuesto por el hecho de que ambas teorías perseguían objetivos distintos y, por ello, no se equiparan en sus términos. Precisamente porque estos autores desarrollan la misma figura lógica en diferentes direcciones, pueden ambos análisis completarse e iluminarse el uno al otro.

Aquí no pretendemos seguir todo el desarrollo conceptual hegeliano con detalle. Para eso, el camino a seguir es tan escarpado como neblinoso. Concretamente lo que se debe estudiar es el hilo conceptual que abarca desde el concepto de vida hasta el concepto de "autoconciencia reconociente», que corresponde en cierta medida al desarrollo desde el concepto de praxis arriba citado hasta el concepto de Estado.

Digámoslo ya: el análisis de Hegel no concibe la autoconciencia como una experiencia psíquica interna. Su tesis principal es justo la contraria, esto es, que la autoconciencia sea una vivencia interna es solamente algo derivado y basado en relaciones externas, en las relaciones sociales. Esta relación es la lucha por el reconocimiento.

Que la autoconciencia se basa en una relación social y no en una conciencia que se define desde dentro, puede ser señalado mediante una analogía con el concepto «mío». Este concepto presupone una frontera entre «mío» y «no mío» que será respetada por los otros. Esta frontera solamente puede ser reconocida como tal por aquellos que se encuentran en el lado de lo "no mío», como una frontera que otro ha puesto y contra la que yo choco. Para poder pensar la exclusión de los otros con respecto a lo «mío», debo primero experimentar mi propia exclusión. Sólo entonces puede ser practicada tal relación de forma inversa.

De forma parecida ocurre con el concepto de Estado. En lo que sigue la idea fundamental es que el Estado no puede entenderse como un ermitaño que primero madura en algún lugar y luego concurre en el mundo, sino que debe entenderse desde el principio como un ser social, el cual se forma completamente a partir y gracias al trato con los otros. Visto desde cerca, el Estado en su forma mas abstracta no es mas que reconocimiento por otros, a saber, reconocimiento

16 Hegel, G. W. F., Fenomenología del Espíritu, Abada Editores, Madrid 2010, Capítulo IV, Autoconciencia. Véase también: Hegel G. W. F., Enciclopedia de las Ciencias Filosóficas, Alianza Editorial, Madrid 2000, Parte 3, §§ 424-436. 
como miembro aceptado en un sistema estatal, lo cual siempre es provisional e inseguro.

Es evidente que estos conceptos están estrechamente relacionados con los conceptos que fueron tratados en relación con la guerra. Las figuras lógicas se comportan aquí de forma análoga, de tal modo que pueden ser traducidos casi mecánicamente. El reconocimiento consiste - también en el razonamiento hegeliano- en el reconocimiento de la capacidad del Estado para poder defenderse a si mismo, para obligar a otros a respetarlo. El reconocimiento recíproco corresponde a la pausa. No obstante, cuando se intentan realizar estas analogías mecánicamente se encuentran algunas dificultades. Estos sistemas están emparentados pero no son idénticos.

La constante disposición para defender su estatus de libre y soberano miembro en una comunidad de Estados es lo que constituye a un Estado. Mediante esta disposición exige el reconocimiento de los otros. La comunidad del Estado no consiste mas que en la sempiterna y recíproca lucha por el reconocimiento, que es fijada en la pausa, o como Hegel lo denominaría, que esta en el estado de Aufhebung (superación incorporadora) $)^{17}$. Por ello el Estado es el resultado de su propia lucha por el reconocimiento, y no sólo sus relaciones externas, sino también las «internas» son determinadas por él. En este nivel del análisis no pueden ser puestas en el concepto de Estado más que estas determinaciones absolutamente abstractas. El punto crucial, la piedra angular es siempre el recíproco reconocimiento (si no se produce supondría un rechazo de reconocimiento, como en la relación de amo y esclavo que Hegel aborda en relación con este tema). Es por esto que el primer rasgo genérico con el que uno se enfrenta en un sistema de Estados, es la igualdad de sus miembros. Esta igualdad es del tipo mas sencilla y consiste en la libertad y soberanía de todos.

Hegel y Clausewitz advierten ambos que la figura lógica es demasiado abstracta, porque los oponentes (aquí los Estados) de esta manera serán sólo espejos idénticos los unos de los otros. Esto significa que los conceptos de diferentes Estados serán idénticos (porque, como ya se ha mencionado, su contenido consiste solamente en su relación con otros conceptos). La separación, que para el concepto de Estado es irrenunciable, se disuelve entonces, y los oponentes se vuelven todos uno.

Aquí tenemos un ejemplo de la hegeliana «identidad de lo opuesto», una contradicción interna, que exige una superación incorporadora. Como esto ocurre en Hegel, no será seguido aquí. Por el contrario, será fructífero comparar qué aspectos tiene esta figura lógica en Clausewitz. Ésta se encuentra en el pasaje sobre la "polaridad» en el primer libro, exactamente en el lugar donde se fundamenta la pausa ${ }^{18}$. En este pasaje habla de repente en puras formas

17 Nota de los traductores: En su traducción de la obra Fenomenología del Espíritu de Hegel, Antonio Gómez Ramos propone traducir el concepto «Aufhebung» según el contexto con las palabras cancelar, asumir o poner en suspenso. Hegel, G. W. F., Fenomenología del Espíritu, traducido por Antonio Gómez Ramos, Abada Editores, Madrid 2010, pp. 973-974.

18 Clausewitz, Carl von, De la guerra, Solar, Buenos Aires 1983, libro 1, capítulo 1, parte 15-16. 
lógicas, completamente diferente que en el resto de la obra. Es evidente que Clausewitz entendió que se había encontrado con un problema lógico de principio, y que había encontrado justamente una respuesta de principio.

Clausewitz constata que los objetivos polares debían ser alcanzados con medios que no se encontrasen en una relación polar los unos con respecto a los otros. Si fueran no solo los objetivos sino también los polos contrarios, esto significaría que las fuerzas que un lado aporta, simultáneamente deben ser una substracción de la misma fuerza del otro lado (exactamente igual que la victoria de aquel que es idéntica a la derrota del otro). En este caso no estaríamos hablando de una guerra entre dos oponentes independientes, puesto que la guerra concerniría a dos espejos idénticos entre si. Los conceptos se fundirían. El combate singular entre dos voluntades, que era el punto de partida de la obra, no carecería solamente del concepto de la pausa, sino que sería el concepto para una lucha entre espejos, una lucha contra sí mismo, y por ello no habría duelo alguno. No habría entonces en el marco del sistema teórico ningún concepto de estrategia, porque la anticipación mutua a los planes del otro es el rasgo de la estrategia: a través de la elección de uno, estaría tomada ya la decisión del otro.

Clausewitz ve claramente cómo la solución debe ser proporcionada de principio. La cuestión debe tratarse de dos cosas diferenciadas entre si, y que tengan una relación común fuera de si, de tal forma que «no estas cosas, sino la relación» sea polar ${ }^{19}$. Como ya se ha mostrado, la solución de Clausewitz consiste realmente solo en una artimaña, en la que introduce conocidas pero indeterminadas concepciones del ataque y la defensa.

Hegel trabaja en el paso de la autoconciencia en si a la autoconciencia reconociente, exactamente con el mismo problema. Los detalles en las conclusiones no son realmente comprensibles, pero evita Hegel la artimaña de Clausewitz, puesto que su construcción total se edifica a partir del concepto de Praxis.

De estas consideraciones se deduce, que el papel que juega el concepto de pausa en Clausewitz es absolutamente central. Es a partir de este concepto que se desarrolla una teoría del conflicto, así como la posibilidad de un concepto de estrategia, que a su vez se basa en algo mas fundamental: a saber, en este punto Clausewitz instituye el concepto de una relación social, una relación entre hombres (o entre Estados) que se comprenden como hombres (Estados) y no como cosas. Surge una relación social porque los espejos se separan y adquieren dimensión independiente. El punto culminante es más claro en el concepto de lucha por el reconocimiento de Hegel, que ha sido medianamente explicado. En este punto Hegel se encuentra entonces, con el concepto del reconocimiento reciproco (esto es, el reconocimiento del reconocimiento de los otros por uno mismo en cuanto reconociente, etc.), la esencia exacta de una relación social: el reconocimiento del otro igual que con el yo. No cabe duda que el reconocimiento recíproco es el núcleo central de la relación estratégica.

19 Ibid., libro 1, capítulo 1, parte 15. 
Lo que hace a un cálculo de posibilidades de actuación un cálculo estratégico es justamente que el objeto de nuestras acciones no se toma como un objeto sin vida, si no que es reconocido como una voluntad autosuficiente, que por sí misma calcula y nos reconoce a nosotros a su vez como estrategas calculantes.

Pero el concepto de la pausa es también central en relación a otro tema. Con la polaridad de los objetivos surge una mera relación «espejo» entre los oponentes. En conexión con el concepto de la pausa se enlaza esa parte «ideal» (los objetivos) con la parte «material» (los medios), a través de una relación de praxis (realmente a partir de dos relaciones de praxis opuestas entre si). Esta parte de los medios es establecida solamente en cierto grado por las relaciones de conceptos, y es por eso que puede adoptar diferentes formas. Esto posibilita no sólo la asimetría entre el ataque y la defensa, sino que también puede suceder que la parte material sea para ambos totalmente distinta. Esto significa que no puede haber solamente una mera relación formal de guerra, sino una relación bélica dentro de un sistema real de fuerzas que se deja determinar en todo momento por fuerzas armadas, vulnerabilidades, etc.

Con la ayuda del concepto de pausa se consigue pues en primer lugar un concepto de Estado, en el cual los Estados no se funden conceptualmente en una identidad, sino que se desligan los unos de los otros, y a través de la superioridad de la defensa son para sí mismos. En segundo lugar se consigue un concepto de Estado que posibilita una gran variedad de Estados (naturalmente diferentes tipos en relación a sus medios de ataque y defensa, puesto que estos Estados hasta ahora sólo se entienden como unidades de combate). El sistema estatal comprende por esto ahora tres rasgos, que más o menos pueden ser descritos de la siguiente manera:

- Igualdad formal de todos los miembros en cuanto igualmente soberanos;

- Desigualdad real, porque sus dominios de soberanía (esto es, lo que la defensa defiende) pueden ser completamente distintos;

- Y acoplamiento de los Estados a través de la guerra, la cual pone los límites en cuanto a qué formación estatal es capaz de sobrevivir (esto es, capaz de defenderse), frente a los otros Estados.

Ya se tiene aquí una sencilla teoría con notable fuerza expresiva. Seria especialmente interesante aplicarla a las primitivas formas estatales, en las cuales el problema de la defensa es presumiblemente decisivo para la forma de los Estados y su organización interna.

Debe ser subrayado, que el concepto de Estado aquí desarrollado no corresponde al concepto hegeliano de Estado. Hegel tiene un concepto propio de Estado, el cual se diferencia del aquí expuesto notablemente, se podría decir que es casi opuesto a este. El no aplica su análisis del reconocimiento y la autoconciencia al Estado (por lo menos no de forma directa y desde el comienzo), sino solamente en relación con los individuos. El Estado hegeliano puede ser descrito en el mejor de los casos como un tipo de autoconciencia general, que de alguna forma surge de la conciencia de los individuos. Ésta por lo tanto se construye desde «dentro». El Estado es constitución, autoridad interna, antes 
de que él participe de cualquier relación con otros Estados ${ }^{20}$. Por ello surgen las ya mencionadas dificultades para comprender la guerra, la paz y el carácter del sistema internacional.

La lucha por el reconocimiento es existencial. Es una lucha a vida o muerte del Estado como Estado (esto es, como reconocido). El reconocimiento en cuanto Estado autónomo - esto es, la soberanía - es la apuesta que se juega en la lucha. Por el contrario, la lucha sólo afecta los procesos vitales dentro del territorio del Estado de forma indirecta. El análisis hegeliano del reconocimiento, trasladado a la relación entre Estados, no desemboca por ello en una masacre, sino en un reconocimiento reciproco o en una relación de dominio y servidumbre. El razonamiento de Clausewitz culmina en el mismo punto: No en el exterminio sino en la pausa o en la victoria sobre la voluntad del oponente, lo que es pasado por alto por aquellos que consideran a Clausewitz como un apóstol de la «guerra total». Ciertamente, después de la victoria se puede tratar al vencido como se quiera (como en la destrucción de Cartago), lo que no pertenece al concepto de guerra en cuanto tal. Es primeramente con las armas atómicas cuando la exterminación es parte del auténtico proceso de la guerra, y desde un punto de vista meramente teórico, lo que constituye la auténtica novedad de las armas atómicas.

La apuesta en la lucha por el reconocimiento es el reconocimiento, esto es, el estatus como miembro de un sistema estatal. Desde un punto de vista material (la lucha), este estatus está condicionado por la capacidad defensiva. Desde un punto de vista ideal (la voluntad), consiste en la capacidad de sostenerse como soberano. Estas solo son naturalmente dos caras de la misma cosa. Es bastante apropiado situar ambas dentro del concepto de «dominio soberano» (aunque tal dominio no debe entenderse necesariamente como territorio). La defensa es la defensa de los dominios soberanos, y la soberanía estatal es la soberanía sobre estos dominios. Este dominio puede ser más o menos amplio. Puede limitarse o ampliarse a través de la guerra, sin embargo si desaparece éste, desaparece entonces el Estado en cuanto Estado. Independientemente de la terminología, se trata de lo mismo cuando Clausewitz habla de la voluntad del Estado.

Análogamente con los anteriores conceptos, el concepto de soberanía debe ser entendido como reflejo y relación social. La soberanía de un Estado sobre un dominio determinado no es en primera línea una relación de este Estado, sino de otros Estados con relación a este dominio. No cabe duda de que la soberna no comprende necesariamente «derecho» o «dominio» sobre este dominio. De esta no se sigue tampoco que el Estado sea capaz de aprovechar dicho dominio (el territorio). Ejercer la soberanía no significa administrar un dominio o intervenir sobre el, sino evitar que otros Estados lo hagan. El dominio soberano es por así decirlo la «objetividad» del Estado, y el reconocimiento

20 Hegel G. W. F., Enciclopedia de las Ciencias Filosóficas, Alianza Editorial, Madrid 2000, Parte 3, §§ 535-547. 
de un Estado consiste únicamente en el reconocimiento de la capacidad de defender un dominio.

Esto muestra de nuevo que el contenido del concepto de Estado no viene de «dentro», sino de «fuera». El Estado no puede ser comprendido como el producto de determinadas instituciones, de una concreta constitución, o de una relación de poder interna, o como algo parecido a esto. Su auténtica esencia no reside en un orden interno y un derecho estatal interno, sino en un derecho externo. Sólo entonces serán comprensibles los rasgos fundamentales de la construcción interna de un Estado, en cuanto efectos de las relaciones en un sistema estatal, como consecuencia de la necesidad de una defensa suficiente. Estos rasgos fundamentales son en primera línea, la paz interna, así como un aparato militar y las condiciones para su subsistencia.

La lucha por el reconocimiento hegeliana se torna visiblemente en algo indeterminado, flotante, simbólico e irrelevante para el bienestar de los hombres. Aquí no se trata de tierras, riqueza o concesiones de actuación, sino del reconocimiento a través de los otros. Se podría opinar por ello (y parece ser también esta la propia visión de Hegel), que la lucha que él estudia se encuentra infinitamente lejos de las luchas del mundo real. Clausewitz sin embargo reconoce desde el principio toda adquisición, considerada en su más absoluta exactitud; no consiste en una posesión sino en una preservación, en una custodia: esto es, en el libre y obligado reconocimiento por los otros. Por esto el objetivo de la guerra es siempre imponer a otros su propia voluntad, y por ello el parentesco entre la lucha por el reconocimiento hegeliana y el concepto de guerra de Clausewitz es tan estrecho.

ANDERs Boserup

BiBLIOGRAFÍA CITADA:

Aron, R., Penser la guerre. Clausewitz, Editions Gallimard, Paris 1976 (español: Pensar la guerra, Clausewitz, Instituto de Publicaciones Navales, Buenos Aires 1988).

Boserup, A., "Krieg, Staat und Frieden. Eine Weiterführung der Gedanken von Clausewitz", en: Weizäcker, Carl Friedrich von (ed.), Die Zukunft des Friedens in Europa. Politische und militärische Voraussetzungen, Hanser, München y Wien 1990, 244-263.

Boserup, A., MAcK, Andrew, War without weapons. Non-Violence in National Defense, Schocken books, New York 1974 (español: Guerra sin Armas. La noviolencia en la defensa nacional. Editorial Fontamara, Barcelona, 1985. Segunda edición: Madrid, Libros de la Catarata, 2001).

Clausewitz, C. von, Vom Kriege. Teil 1-3. Ferdinand Dümmler, Berlin 1831-1833 (español: Clausewitz, Carl von, De la guerra, Solar, Buenos Aires, 1983).

Coumier, Y., "Hegel and Clausewitz: Convergence on Method, Divergence on Ethics". The International History Review 36 (3), 2014, pp. 419-442. 
Hegel, G. W. F. (1830), Enzyklopädie der philosophischen Wissenschaften. Berlin.

(español: Enciclopedia de las Ciencias Filosóficas; Edición, introducción y notas de Ramón Valls Plana, Alianza Editorial, Madrid 2000).

Hegel, G. W. F., Phänomenologie des Geistes. Verlag Joseph Anton Goebhardt, Bamberg, Würzburg 1807 (español: Hegel, G. W. F., Fenomenología del Espíritu, Abada Editores, Madrid 2010).

Herberg-Rothe, A., "Clausewitz und Hegel. Ein heuristischer Vergleich". Forschungen zur brandenburgischen und preußischen Geschichte, 10 (1) 2000, pp. 49-84.

Rothe, B., Türpe, A., "Das Wesen des Krieges bei Hegel und Clausewitz”, Deutsche Zeitschrift für Philosophie 25, 1977, pp. 1331-1343.

Villacañas Berlanga, J. L., La Nación y la Guerra. Confederación y hegemonía como formas de concebir Europa, Editum, Murcia, 1999.

[Escrito aprobado para su publicación en diciembre de 2015] 